\title{
Manfred Schedlowski: «Gezielte Verhaltens- interventionsprogramme können das biochemische Netzwerk im Körper beeinflussen»
}

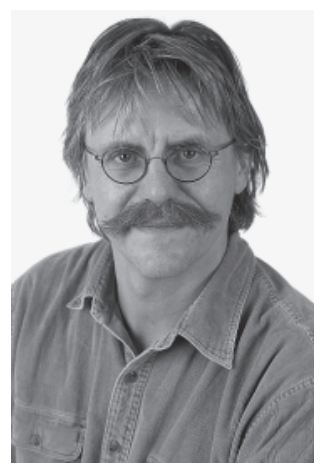

Manfred Schedlowski ist Professor für Medizinische Psychologie und Verhaltensneurobiologie am Universitätsklinikum in Essen. Als Klinischer Psychologe beschäftigt er sich mit den funktionellen Zusammenhängen zwischen dem Gehirn, dem neuroendokrinen System und dem Immunsystem. Er untersucht einerseits in tierexperimentellen Studien und andererseits im Humanbereich an gesunden Probanden und Patienten die Auswirkungen von Verhalten sowie Stressexpositionen, Verhaltensinterventionen und Konditionierungsprozessen auf das körpereigene Abwehrsystem und auf die Entstehung und den Verlauf von Erkrankungen. Im deutschsprachigen Raum ist er einer der führenden Experten der Psychoneuroimmunologie. Die Fragen stellte Ulrike Ehlert, Professorin für Klinische Psychologie und Psychotherapie an der Universität Zürich.
Wo liegen die bahnbrechenden Erkenntnisse der differentialdiagnostischen Psychoneuroimmunologie?

Manfred Schedlowski: Zunächst einmal zeigen die experimentellen Befunde der Psychoneuroimmunologie aus den letzten 15-20 Jahren, dass das Immunsystem, ähnlich wie auch das Hormonsystem oder Herz-Kreislauf-Funktionen, sensitiv auf Verhaltenseinflüsse reagiert. Heute wissen wir, dass akuter und chronischer Stress beispielsweise die Zirkulation von immunkompetenten Zellen im Körper nachhaltig verändern kann. Das betrifft nicht nur die funktionelle Kapazität dieser Zellen, also wie gut die einzelnen Zellen ihrer Aufgabe gewachsen sind, sondern auch eine Reihe von Immunbotenstoffen, die Zytokine, die die Immunabwehr gegen Viren oder Bakterien, entartete Zellen oder entzündliche Prozesse steuern. Das sind extrem wichtige Befunde, weil sie die lange bestehende Sichtweise verändert haben, dass das Immunsystem im Körper autonom gesteuert wird und nur durch eindringende Pathogene aktiviert werden kann.
Für welche Störungsbilder und körperlichen Erkrankungen ist die Berücksichtigung psychoneuroimmunologischer Befunde unabdingbar?

Manfred Schedlowski: Von vielen Erkrankungen, an denen das Immunsystem beteiligt ist, wissen wir aus der klinischen Praxis, dass insbesondere belastende psychische Ereignisse zu einer Verschlechterung der Krankheitssymptome führen. Psychoneuroimmunologische Befunde zeigen, dass bei Patienten, zum Beispiel mit chronischen Autoimmunerkrankungen wie Polyarthritis, entzündlichen Darmerkrankungen oder Hauterkrankungen, die psychoneuroimmunologische Kommunikation auf dem efferenten Weg vom Zentralen Nervensystem zum peripheren Immunsystem gestört zu sein scheint. Es kommt an unterschiedlichen Schaltstellen zu «Übertragungsfehlern». Mit Schaltstellen meine ich einerseits das Gehirn, unser Stress wahrnehmendes Organ, andererseits das neuroendokrine System, das die Stresssignale zum peripheren Immunsystem weiterleitet, und schließlich die Organe und

\begin{tabular}{llll}
\hline KARGER & $\oplus$ ( 2007 S. Karger GmbH, Freiburg & & Prof. Dr. Manfred Schedlowski \\
Fax +49 761 4 520714 & Accessible online at: & Untitut für Medizinische Psychologie und Verhaltensimmunbiologie \\
E-mail Information@Karger.de & www.karger.com/ver & Hufelandstr. 55, \\
www.karger.com & & 45122 Essen, Deutschland \\
& & E-mail manfred.schedlowski@uk-essen.de
\end{tabular}


Zellen des Immunsystems. Bei diesen Autoimmunerkrankungen entstehen die «Übertragungsfehler» zum Beispiel auf der Rezeptorebene, wodurch das bei diesen Patienten ohnehin schon belastete Immunsystem durch Stress noch weiter aus dem Gleichgewicht gebracht wird. Dies führt schließlich zur Exazerbation der Krankheitssymptome. Hier besteht allerdings noch großer Forschungsbedarf, um die Abläufe dieser biochemischen Signalkaskaden detailliert weiterzuanalysieren. Denn nur auf der Grundlage solcher genauer Erkenntnisse lassen sich dann auch gezielte nichtpharmakologische Interventionsprogramme entwickeln und einsetzen.

Bei psychischen und psychiatrischen Störungsbildern wie depressiven oder schizophrenen Erkrankungen mehren sich die Befunde, die dabei eine eindeutige Rolle von löslichen Immunbotenstoffen dokumentieren. Das heißt, dass Zytokine in der Pathophysiologie dieser Erkrankungen beteiligt sind. Bei diesen Patienten scheint der afferente Weg vom peripheren Immunsystem zum ZNS gestört zu sein. Insbesondere bei den depressiven Erkrankungen scheinen ähnliche Prozesse zu wirken, wie wir sie alle unter dem Begriff der unspezifischen Krankheitssymptome (sickness behavior) kennen und selbst wahrscheinlich schon oft durchlebt haben. Bei einem aufkommenden grippalen Infekt fühlen wir uns oft schon Tage vor Ausbruch der eigentlichen Erkrankung und dem Auftreten der körperlichen Symptomatik müde, antriebslos und oft auch depressiv verstimmt. Diese unspezifischen Krankheitssymptome werden durch eine aktivierte Immunabwehr verursacht, die als Antwort auf die Pathogene vermehrt so genannte proinflammatorische Zytokine produziert und ausschüttet. Diese modulieren dann über bisher noch nicht klar erkannte Mechanismen funktionelle Prozesse im Zentralen Nervensystem.

Inwieweit berücksichtigt heute die Onkologie die Erkenntnisse der Psychoneuroimmunologie?

Manfred Schedlowski: In der Psychoonkologie gibt es spätestens seit der initialen Arbeit von David Spiegel aus dem Jahr 1989, die im «Lancet» publiziert wurde, eine kontrovers geführte Diskussion. Es wird immer wieder erörtert, ob und inwieweit das Immunsystem onkologischer Patienten durch Verhaltensinterventionen positiv beeinflusst werden kann. Das heißt, es wird geprüft, ob durch Verhaltensinterventionen karzinogene Prozesse gehemmt und dadurch das Überleben der Patienten verlängert werden kann.

Die Befundlage aus den bisher publizierten Studien reflektiert diese Kontroverse insoweit, als etwa die Hälfte der Untersuchungen einen positiven Effekt solcher Verhaltensinterventionen auf immunologische Funktionen bzw. die Überlebenszeit der Patienten berichtet. Die andere Hälfte kann jedoch keine Effekte dokumentieren. Diese Befundlage mag aber auch darin begründet sein, das viele Tumore und karzinogene Prozesse wie die Metastasierung gar nicht oder nur über kurze Phasen vom Immunsystem kontrolliert werden. Hierzu wurden Ende letzten Jahres ganz neue tierexperimentelle
Erkenntnisse von einer amerikanischen Arbeitsgruppe in «Nature Medicine» publiziert. Diese Ergebnisse zeigen, dass die Tumorentstehung und die Metastasierung von Tumoren maßgeblich direkt durch die bei Stress ausgeschütteten Hormone Adrenalin und Noradrenalin und durch die entsprechenden Rezeptoren auf den Tumorzellen gesteuert werden. An diesen Vorgängen waren keine immunkompetenten Zellen beteiligt.

Inwiefern gewinnt die Psychotherapie durch psychoneuroimmunologische Diagnostik und Evaluation?

Manfred Schedlowski: Die funktionellen psychoneuroimmunologischen Zusammenhänge bei Erkrankungen mit immunologischen Bezug werden in immer mehr Einzelheiten bekannt. Auf der Grundlage dieser Erkenntnisse wird es notwendig sein, gezielte Verhaltensinterventionsprogramme zu gestalten, mit denen sich auf das biochemische Netzwerk im Körper Einfluss nehmen lässt. Beispielsweise wurde gerade in den letzten Jahren detailliert dokumentiert, dass Bewegung und Sport antientzündlich wirken. Insbesondere antiinflammatorische Zytokine werden durch sportliche Aktivitäten vermehrt im Körper freigesetzt. Diese neuen Erkenntnisse haben dazu geführt, dass Sport und Bewegung bei einer Vielzahl von Erkrankungen als nichtpharmakologische Behandlungsmethode unter dem Überbegriff «Die Heilkraft der Bewegung» eingesetzt werden.

Inzwischen wissen wir aus vielen Grundlagenstudien der Placebo- und der Konditionierungsforschung, dass auch Immunfunktionen beim Menschen klassisch konditioniert werden können. Das bedeutet, dass im Rahmen von künftigen Verhaltensinterventionsprogrammen Erwartungseffekte und Konditionierungsprozesse gezielt als additive oder supportive Therapie zur pharmakologischen Intervention eingesetzt werden sollten. Klares Ziel ist es dabei, Medikamente und damit unerwünschte Nebenwirkungen einzusparen und gleichzeitig die therapeutischen Effekte zu maximieren.

Gibt es immunologische Parameter, die auch in der alltäglichen psychotherapeutischen Praxis gemessen werden können? Braucht es hierfür die Kooperation mit Fachärzten?

Manfred Schedlowski: Die Bestimmung der immunologischen Parameter ist bisher noch recht schwierig und spezialisierten Laboren vorbehalten. Meistens sind auch Facharztpraxen nicht mit der dafür notwendigen Laborlogistik ausgestattet. Allerdings geht die Entwicklung von einfachen und kostengünstigen Methoden zur Bestimmung von beispielsweise Zytokinen in einem rasanten Tempo voran. Es ist daher sehr wahrscheinlich, dass man solche Bestimmungen mit einfachen Test-Kits in einigen Jahren auch routinemäßig und ohne große Laborerfahrung durchführen kann. Allerdings wird die Interpretation der Befunde natürlich von dem zugrunde liegenden Wissen um die Bedeutung dieser Parameter im Rahmen der 
jeweiligen Erkrankung abhängig sein und natürlich auch vom Kenntnisstand des jeweiligen Untersuchers oder der jeweiligen Untersucherin.

Wie können sich praktisch arbeitende Psychotherapeuten am besten in das Gebiet der Psychoneuroimmunologie einarbeiten?

Manfred Schedlowski: In der deutschsprachigen Literatur gibt es bis auf ein älteres Lehrbuch zur Psychoneuroimmunologie [Schedlowski und Tewes, 1996] immer noch zu wenig einschlägige Lehrbücher und Texte, die eine Einarbeitung in dieses Forschungsgebiet erleichtern. Neu erschienen ist unter anderem ein Lehrbuch zur Klinischen Pathophysiologie kom- plexer Erkrankungen [Straub, 2006], in dem insbesondere psychoneuroimmunologische Aspekte bei unterschiedlichen Erkrankungen im Vordergrund stehen; weitere einleitende Texte zur Psychoneuroimmunologie finden sich auch bei Ehlert [2003].

\section{Literatur}

Ehlert U (Hrsg): Verhaltensmedizin. Heidelberg, Springer, 2003

Schedlowski M, Tewes U (Hrsg): Psychoneuroimmunologie. Heidelberg, Spektrum, 1996.

Straub RH (Hrsg): Lehrbuch der klinischen Pathophysiologie komplexer chronischer Erkrankungen in 2 Bänden. Band 1: Physiologische Grundlagen. Band 2: Spezielle Pathophysiologie. Göttingen, Vandenhoeck und Ruprecht, 2006. 